\title{
Um estudo de genética quantitativa sobre agregação familiar na composição corporal de famílias nucleares portuguesas
}

\author{
Rogério C. Fermino \\ André Seabra \\ Rui Garganta \\ Alcibíades B. Valdivia \\ José Maia
}

https://doi.org/10.5628/rpcd.08.01.77

\author{
Laboratório de Cineantropometria e \\ Gabinete de Estatística Aplicada \\ Faculdade de Desporto \\ Universidade do Porto \\ Portugal
}

\section{RESUMO}

Este estudo teve como objectivo (1) verificar a presença indirecta de transmissão vertical de factores genéticos entre progenitores e descendentes nos fenótipos da composição corporal e (2) estimar a contribuição dos factores genéticos responsáveis pela variação nos fenótipos da composição corporal em termos populacionais.

A amostra foi constituída por 363 indivíduos pertencentes a 107 famílias nucleares participantes do projecto FAMÍLIAS ACTIVAS. Os fenótipos da composição corporal foram avaliados com um aparelho de impedância bioeléctrica da marca Tanita ${ }^{\circledR}$ modelo BC-418MA. Foi utilizado o software PEDSTATS para analisar o comportamento genérico das variáveis entre os diferentes membros da família. O cálculo das correlações entre familiares e as estimativas de heritabilidade foram realizados nos módulos FCOR e ASSOC do software de Epidemiologia Genética S.A.G.E. versão 5.3

Os valores dos coeficientes de correlação entre os graus de parentesco foram baixos a moderados $(-0,04 \leq r \leq 0,65)$. Os factores genéticos explicaram entre 35 a $46 \%$ da variação dos diferentes fenótipos da composição corporal, sendo a maior contribuição verificada para a quantidade absoluta de gordura corporal $(43 \%)$ e a massa muscular $(46 \%)$.

Estes resultados indicam uma forte agregação familiar na composição corporal nesta amostra de famílias nucleares portuguesas.

Palavras-Chave: agregação familiar, heritabilidade, composição corporal, epidemiologia genética, famílias nucleares.

\section{ABSTRACT \\ A quantitative genetic study about familial aggregation in body composition of portuguese nuclear families}

This study aims (1) to verify the indirect presence of vertical transmission of genetic factors between parents and offspring and (2) to estimate the contribution of the genetic factors in the variance of different phenotypes describing body composition.

Sample size comprises 363 subjects from the 107 nuclear families participating in the project "FAMILIAS ACTIVAS". Body composition phenotypes were measured with a bioelectric impedance device Tanita ${ }^{\circledR}$ model BC-418MA. PEDSTATS software was used to verify the structure of each family and to analyze the generic behavior of the phenotypes between the different members of the family. Familiar correlations and heritability estimates $\left(h^{2}\right)$ were computed in the FCOR and ASSOC modules of S.A.G.E. 5.3 software.

Correlation coefficients between relatives were low to moderate $(-0,04 \leq$ $r \leq 0,65)$. Genetic factors explained between $35-46 \%$ of different body composition phenotypes. The largest contributions were related to total body fat (43\%) and lean body mass (46\%)

These results showed an important familial aggregation in body composition values of Portuguese nuclear familiar samples.

Key-words: familial aggregation, heritability, body composition, Genetic Epidemiologic, nuclear families 


\section{INTRODUÇÃOO}

A composição corporal (CC) é uma das componentes da aptidão física relacionada com a saúde ${ }^{(1)}$ e a determinação de seus vários fenótipos possui aplicações em diversas áreas das Ciências do Desporto(7). É corrente o seu uso em estudos de intervenção ou de ensaios clínicos sobre a actividade física cujo propósito é verificar as alterações em diferentes factores de risco de diversas morbilidades (7).

A CC pode ser estimada com técnicas laboratoriais e de terreno que variam em termos de complexidade, custo e precisão(1,7). Algumas das técnicas laboratoriais disponíveis, e mais utilizadas, são a densitometria computorizada por absorciometria radiológica de dupla energia (DEXA), a pesagem hidrostática e a tomografia computorizada. Dentre as técnicas de terreno, as mais utilizadas são a medição de prega de adiposidade subcutânea e a impedância bioelétrica (BIA) ${ }^{(7,14)}$. Os sistemas de BIA estão sendo cada vez mais utilizados tanto no meio clínico como no científico(21), devido ao facto de não serem invasivos(7), terem elevada portabilidade, serem relativamente baratos e não exigirem treino específico do avaliador(7,14).

Uma das questões fundamentais no estudo da gordura corporal (GC) é a sua distribuição, nomeadamente no que se refere à adiposidade peri-visceral, visto estar descrita a sua associação com o risco elevado de manifestação de determinadas doenças crónicas(31), tais como: a hipertensão arterial, a doença arterial coronária, a dislipidemia e a diabetes tipo $11(4,10)$. Para além disso, o sobrepeso e a obesidade são importantes factores de risco para o desenvolvimento de doenças cardiovasculares(2) que apresentam uma elevada prevalência em todas as faixas etárias e vem aumentando nos últimos anos(17).

Recentemente, a American Heart Association(2) publicou dados epidemiológicos acerca do elevado impacto financeiro que a epidemia da obesidade representa para a sociedade. Sabe-se também que tanto factores genéticos quanto ambientais estão envolvidos na etiologia desta doença $(4,31)$ e, por isso, a obesidade é considerada uma doença poligénica e multifactorial, dada a complexidade de factores responsáveis pela sua manifestação.

A influência familiar, em um determinado fenótipo, pressupõe a interacção entre factores genéticos e ambientais. Existe forte evidência de informação proveniente de pesquisas em Epidemiologia Genética que refere a importância do ambiente familiar na variabilidade nos indicadores da CC. Essa influência é resultante da partilha de genes e do envolvimento comum pelos membros da mesma família( ${ }^{(6)}$. Diversos estudos $(11,13,16,19,23,25,27,31)$ mostraram que tanto os genes quanto o ambiente, partilhado entre membros da família, contribuem para a agregação familiar (AgF) na quantidade de GC relativa $\left(\mathrm{GC}_{\text {rel }}\right)$ e absoluta $\left(\mathrm{GC}_{\mathrm{abs}}\right)$, na gordura subcutânea e na massa isenta de gordura (MIG). Com base em estudos familiares para verificar AgF, estimou-se que cerca de $30^{(11)}$ a $76 \%$ (27) da variação total dos valores de diferentes indicadores da CC pode ser atribuída a diferenças genéticas entre sujeitos, ficando o restante a dever-se aos factores ambientais.

Não parece existir muita informação oriunda da Epidemiologia Genética afim de analisar a importância dos factores genéticos, responsáveis pela variabilidade dos valores do fraccionamento de diferentes segmentos corporais, com base em informação providenciada pela BIA. No espaço Lusófono não são conhecidos estudos com este tipo de abordagem. Face à inexistência de informações disponíveis em língua portuguesa acerca dos aspectos de $\mathrm{AgF}$ na CC, estabelecemos os seguintes objectivos: (1) verificar a presença indirecta de transmissão vertical de factores genéticos entre progenitores e descendentes nos fenótipos da CC; (2) estimar a contribuição dos factores genéticos responsáveis pela variação nos fenótipos da $\mathrm{CC}$ em termos populacionais.

\section{MATERIAL E MÉTODOS}

\section{Amostra}

O projecto FAMÍLIAS ACTIVAS tem, numa primeira instância, o propósito de estudar e referenciar aspectos genéticos e ambientais na actividade física, aptidão física, componentes da síndrome metabólica (SM), hábitos nutricionais e factores comportamentais de risco em famílias nucleares. Na segunda etapa lidará com aconselhamento e intervenção junto das famílias com o propósito de alterar comportamentos e hábitos de risco. A amostragem deste projecto está dividida por diferentes locais com base no voluntariado de crianças e jovens que desejam 
envolver a sua família nesta pesquisa, desde que tenham pelo menos um irmão ou irmã com mais de sete anos de idade.

Os dados utilizados referem-se ao estudo piloto da primeira fase do projecto. Para este fim, contactámos as escolas onde tivemos maior facilidade de acesso em alguns distritos na região Norte do país afim de verificar a possível adesão ao projecto. Nos locais de melhor receptividade, enviámos uma comunicação escrita a cada família convidando-a a participar na pesquisa. No texto enviado havia uma explicação breve acerca do propósito do estudo, bem como informação relativa ao consentimento de participação. Após obtenção do consentimento informado devidamente assinado, foi enviada outra comunicação às famílias explicando detalhadamente os procedimentos necessários para a realização da recolha dos dados, bem como o agendamento da data, horário e local. Os indivíduos foram avaliados no período matutino e em jejum. Foram excluídos os seguintes casos: (1) pais e/ou mães não biológicos (um pai) e (2) indivíduos que não estavam em jejum (três mães e uma filha).

A amostra foi constituída por 363 indivíduos (161 progenitores - 40,1 $\pm 4,5$ anos e 202 descendentes 13,3 \pm 3 anos) pertencentes a 107 famílias nucleares (Tabela 1). Contudo, em apenas 58 famílias ambos os progenitores compareceram na recolha de dados.

\section{Mensuração dos indicadores da composição corporal}

Para a avaliação da CC foi utilizado um aparelho de BIA da marca Tanita ${ }^{\circledR}$ modelo BC-418MA (Tanita Corp., Tokyo, Japan). Esse dispositivo possui quatro eléctrodos nas mãos (palmas e dedos) e quatro nos pés (calcanhares e regiões plantares). No total, cinco segmentos são medidos (membros inferiores, membros superiores e tronco). No output constam, entre outras variáveis, a quantidade de GC, gordura no tronco (GT), gordura nos membros (GM), MIG e massa muscular (MM) expressas em termos relati$\operatorname{vos}\left({ }_{\text {rel }}\right)$ e absolutos $($ abs $)$. Quando comparada a DEXA, a CC medida por este aparelho apresenta correlações elevadas tanto para análises segmentais $\left(\mathrm{GC}_{\mathrm{rel}}=0,79-0,85\right.$ e $\left.\mathrm{MIG}_{\mathrm{abs}}=0,95-0,96\right)$ quanto de corpo inteiro $\left(\mathrm{GC}_{\mathrm{rel}}=0,89 \text { e } \mathrm{MIG}_{\mathrm{abs}}=0,96\right)^{(21)}$.

A estatura foi medida com um antropómetro portátil da marca Siber Hegner ${ }^{\circledR}$ com precisão de 0,1 cm. O antropómetro foi fixado numa base de madeira confeccionada para tal finalidade. $\mathrm{O}$ indivíduo descalço posicionava-se de pé, com os calcanhares apoiados na referida base, glúteos e o dorso apoiados no antropómetro e cabeça posicionada no plano de Frankfurt. A estatura foi medida entre o vertex e o plano de referência da base de madeira(12).

A massa corporal foi medida no mesmo aparelho de BIA que possui precisão de $0,1 \mathrm{~kg}$. O indivíduo deveria estar na posição antropométrica de referência $^{(12)}$, descalço e vestindo roupas leves.

$\mathrm{O}$ índice de massa corporal (IMC) foi então calculado dividindo-se o peso $(\mathrm{kg})$ pela estatura $(\mathrm{m})$ ao quadrado, obtendo um valor final expresso em $\mathrm{kg} / \mathrm{m}^{2}$.

\section{Análise Estatística}

O software estatístico SPSS 15.0 foi utilizado na análise exploratória de dados afim de verificar possíveis erros de entrada das informações, a presença de outliers e a normalidade das distribuições, bem como para calcular a média, o desvio padrão e a amplitude das variáveis. $\mathrm{O}$ t-teste de medidas independentes foi aplicado para verificar diferenças nas médias das variáveis entre os grupos. Foi utilizado o software PEDSTATS(30) para inspeccionar a estrutura de cada família e analisar o comportamento genérico das variáveis entre os diferentes membros da família. Para verificar AgF e calcular as estimativas de heritabilidade $\left(h^{2}\right)$, foram utilizados os módulos FCOR e ASSOC do software de Epidemiologia Genética S.A.G.E. 5.3(26). Todos os fenótipos considerados foram ajustados às covariáveis idade, sexo, idade ${ }^{2}$, idade $^{3}$, idade $x$ sexo, idade $2 x$ sexo e IMC. Foi adoptado o nível de significância de 0,05.

\section{RESULTADOS}

Em média, ambos os progenitores apresentaram valores de IMC semelhantes, evidenciando sobrepeso $\left(\geq 25 \mathrm{~kg} / \mathrm{m}^{2}\right)$. Com excepção da quantidade relativa de gordura nos membros $\left(\mathrm{GM}_{\mathrm{rel}}\right)$, as mães apresentaram valores significativamente superiores nos demais fenótipos relacionados a quantidade $\left(\mathrm{GC}_{\text {rel }}\right.$ e $\mathrm{GC}_{\mathrm{abs}}$ ) e distribuição de gordura (GT). Nos fenótipos $\mathrm{MIG}_{\mathrm{rel}}, \mathrm{MM}_{\mathrm{rel}}$ e $\mathrm{MM}_{\mathrm{abs}}$ o comportamento foi inverso. Para os descendestes, este dimorfismo foi semelhante em todos os fenótipos (Tabela 1). 
Tabela 1. Medidas descritivas das variáveis da amostra.

\begin{tabular}{|c|c|c|c|c|c|}
\hline \multirow[t]{3}{*}{ Variáveis } & \multicolumn{4}{|c|}{ Progenitores } & \multirow[b]{3}{*}{$p$} \\
\hline & \multicolumn{2}{|c|}{ Pais $[n=64$ ] } & \multicolumn{2}{|c|}{ Mães [n=97] } & \\
\hline & $m \pm d p$ & amplitude & $m \pm d p$ & amplitude & \\
\hline Idade (anos) & $42,5 \pm 4,3$ & $33-56$ & $39,9 \pm 4,3$ & $30-53$ & $<0,001$ \\
\hline Massa corporal (kg) & $77,1 \pm 11,4$ & $52,8-119,2$ & $68,3 \pm 11,8$ & $47,2-103$ & $<0,001$ \\
\hline Estatura $(\mathrm{cm})$ & $168,2 \pm 5,6$ & $155,6-185,5$ & $156,9 \pm 5,2$ & $144,2-171,6$ & $<0,001$ \\
\hline IMC (kg/m2] & $27,2 \pm 3,7$ & $18,7-42,6$ & $27,8 \pm 5$ & $20-43,4$ & 0,379 \\
\hline GCrel $[\%]$ & $22,2 \pm 5$ & $8,1-39,2$ & $33,7 \pm 6,5$ & $18-50,5$ & $<0,001$ \\
\hline GCabs (kg) & $17,5 \pm 6,3$ & $4,3-46,7$ & $23,7 \pm 8,5$ & $9,3-52$ & $<0,001$ \\
\hline GTrel (\%) & $23,7 \pm 5,6$ & $6,6-40,2$ & $29 \pm 7,3$ & $11,7-47,1$ & $<0,001$ \\
\hline GMrel [\%] & $76,3 \pm 5,6$ & $59,8-93,4$ & $71 \pm 7,3$ & $52,9-88,3$ & $<0,001$ \\
\hline MIGrel (\%) & $77,8 \pm 5$ & $60,8-91,9$ & $66,3 \pm 6,7$ & $49,5-82,1$ & $<0,001$ \\
\hline MMrel (\%) & $74,1 \pm 5,3$ & $57,5-88,1$ & $62,9 \pm 6,2$ & 47,1 - 78 & $<0,001$ \\
\hline MMabs (kg) & $56,7 \pm 6,2$ & $43-72,9$ & $42,4 \pm 4,2$ & $33,3-56,5$ & $<0,001$ \\
\hline \multirow[t]{3}{*}{ Variáveis } & \multicolumn{4}{|c|}{ Descendentes } & \\
\hline & \multicolumn{2}{|c|}{ Filhos $(n=81)$} & \multicolumn{2}{|c|}{ Filhas ( $n=121$ ) } & \\
\hline & $m \pm d p$ & amplitude & $m \pm d p$ & amplitude & $p$ \\
\hline Idade (anos) & $12,8 \pm 2,7$ & $7-19$ & $13,5 \pm 3,2$ & $7-25$ & 0,137 \\
\hline Massa corporal (kg) & $50,7 \pm 18,2$ & $20,3-114,7$ & $50,4 \pm 12,4$ & $22,9-92$ & 0,908 \\
\hline Estatura $(\mathrm{cm})$ & $155,3 \pm 15,7$ & $104,4-181$ & $154,1 \pm 9,6$ & $119-169,5$ & 0,477 \\
\hline IMC (kg/m2] & $20,4 \pm 4,4$ & $12,6-38,5$ & $21 \pm 3,8$ & $13,8-35,8$ & 0,294 \\
\hline GCrel $(\%)$ & $19,1 \pm 5,8$ & $11,5-41,9$ & $25,9 \pm 5,4$ & $17,2-45,3$ & $<0,001$ \\
\hline GCabs (kg] & $10,1 \pm 6,6$ & $2,7-48,1$ & $13,5 \pm 5,9$ & $4,3-41,6$ & $<0,001$ \\
\hline GTrel (\%) & $14,8 \pm 6$ & $6,5-39,1$ & $19,8 \pm 6,1$ & $10-43,3$ & $<0,001$ \\
\hline GMrel [\%] & $85,2 \pm 6$ & $60,9-93,5$ & $80,2 \pm 6,1$ & $56,7-90$ & $<0,001$ \\
\hline MIGrel (\%) & $80,9 \pm 5,9$ & $58,1-88,6$ & $74,1 \pm 5,4$ & $54,8-82,9$ & $<0,001$ \\
\hline MMrel [\%] & $77,7 \pm 5,7$ & $55,4-85,2$ & $70,5 \pm 5,2$ & $52,1-78,9$ & $<0,001$ \\
\hline MMabs (kg) & $38,9 \pm 12,7$ & $17-66,2$ & $35,1 \pm 6,9$ & $17,8-48,8$ & 0,005 \\
\hline
\end{tabular}

IMC: índice de massa corporal, m: média, dp: desvio padrão, GCrel: gordura corporal relativa, GCabs: gordura corporal absoluta, GTrel: gordura no tronco relativa, GMrel: gordura nos membros relativa, MIGrel: massa isenta de gordura relativa, MMrel: massa muscular relativa, MMabs: massa muscular absoluta, p: valor de prova.

A Tabela 2 apresenta as correlações entre familiares e as estimativas de $\mathrm{h}^{2}$ para os sete fenótipos da CC. Entre cônjuges, as correlações foram baixas para todos os fenótipos $(-0,04 \leq r \leq 0,23)$. Na relação entre progenitores-descendentes, os valores dos coeficientes de correlação $(r)$ situaram-se entre $-0,03$ a 0,48. Os valores mais expressivos foram encontrados na relação entre pai-filho para a $\mathrm{GC}_{\mathrm{abs}}(r=0,45)$ e entre pai-filha para a $\mathrm{MM}_{\mathrm{abs}}(r=0,48)$. Entre descendentes, as correlações foram positivas, contudo, apresentaram magnitudes diferenciadas. O menor e o maior valor foram verificadas entre irmão-irmão para a
$\mathrm{GM}_{\text {rel }}(r=0,01)$ e $\mathrm{GC}_{\mathrm{abs}}(r=0,65)$, respectivamente. As estimativas de $h^{2}$ encontradas foram moderadas $(0,35-0,46)$ e estatisticamente significativas. Os valores mais elevados foram verificados para a $\mathrm{GC}_{\mathrm{abs}}$ e $\mathrm{MM}_{\mathrm{abs}}(0,43$ e 0,46, respectivamente).

\section{DISCUSSÃO}

Os resultados encontrados mostraram que nos fenótipos relacionados a GC, os indivíduos do sexo feminino apresentaram valores médios significativamente superiores (excepto para $\mathrm{GM}_{\text {rel }}$ ). De certa maneira, houve uma certa dificuldade na comparação directa 
Tabela 2. Coeficientes de correlação ( $r \pm$ erro padrão) entre familiares e estimativas de $h^{2}$ para os fenótipos da composição corporal.

\begin{tabular}{|c|c|c|c|c|c|c|c|c|}
\hline $\begin{array}{l}\text { Grau de } \\
\text { parentesco }\end{array}$ & $\begin{array}{l}\mathrm{n}^{0} \mathrm{de} \\
\text { pares }\end{array}$ & $\begin{array}{l}\text { GCrel* } \\
r \pm e p\end{array}$ & $\begin{array}{c}\text { GCabs } † \\
r \pm e p\end{array}$ & $\begin{array}{l}\text { GTrel* } \\
r \pm e p\end{array}$ & $\begin{array}{l}\text { GMrel* }^{*} \\
r \pm e p\end{array}$ & $\begin{array}{c}\text { MIGrel* }^{*} \\
r \pm e p\end{array}$ & $\begin{array}{c}\text { MMrel* }^{*} \\
r \pm e p\end{array}$ & $\begin{array}{c}\text { MMabs } \neq \\
r \pm e p\end{array}$ \\
\hline Cônjuges & 58 & $-0,14 \pm 0,13$ & $0,16 \pm 0,13$ & $-0,15 \pm 0,13$ & $-0,15 \pm 0,13$ & $-0,14 \pm 0,13$ & $-0,04 \pm 0,13$ & $0,23 \pm 0,13$ \\
\hline Pai - filho & 53 & $0,20 \pm 0,14$ & $0,45 \pm 0,12$ & $0,20 \pm 0,14$ & $0,20 \pm 0,13$ & $0,20 \pm 0,14$ & $0,23 \pm 0,14$ & $0,37 \pm 0,12$ \\
\hline Pai - filha & 75 & $-0,04 \pm 0,13$ & $0,20 \pm 0,12$ & $-0,04 \pm 0,13$ & $-0,03 \pm 0,13$ & $-0,04 \pm 0,13$ & $0,03 \pm 0,13$ & $0,48 \pm 0,10$ \\
\hline Mãe - filho & 74 & $0,13 \pm 0,12$ & $0,35 \pm 0,11$ & $0,14 \pm 0,12$ & $0,14 \pm 0,12$ & $0,12 \pm 0,12$ & $0,11 \pm 0,12$ & $0,14 \pm 0,12$ \\
\hline Mãe - filha & 117 & $0,25 \pm 0,09$ & $0,13 \pm 0,09$ & $0,23 \pm 0,09$ & $0,24 \pm 0,09$ & $0,24 \pm 0,09$ & $0,25 \pm 0,09$ & $0,22 \pm 0,10$ \\
\hline Irmão - irmão & 11 & $0,25 \pm 0,30$ & $0,65 \pm 0,18$ & $0,04 \pm 0,31$ & $0,01 \pm 0,32$ & $0,22 \pm 0,30$ & $0,15 \pm 0,31$ & $0,16 \pm 0,31$ \\
\hline Irmã - irmã & 34 & $0,28 \pm 0,16$ & $0,12 \pm 0,17$ & $0,22 \pm 0,17$ & $0,23 \pm 0,17$ & $0,29 \pm 0,16$ & $0,32 \pm 0,16$ & $0,30 \pm 0,16$ \\
\hline Irmão - irmã & 59 & $0,12 \pm 0,14$ & $0,14 \pm 0,13$ & $0,07 \pm 0,14$ & $0,07 \pm 0,14$ & $0,11 \pm 0,14$ & $0,10 \pm 0,14$ & $0,10 \pm 0,14$ \\
\hline$h^{2}$ & & $\begin{array}{l}0,37 \pm 0,12 \\
(p<0,001)\end{array}$ & $\begin{array}{l}0,43 \pm 0,10 \\
(p<0,001)\end{array}$ & $\begin{array}{l}0,35 \pm 0,12 \\
(p=0,002)\end{array}$ & $\begin{array}{l}0,36 \pm 0,12 \\
(p=0,002)\end{array}$ & $\begin{array}{l}0,35 \pm 0,12 \\
(p=0,002)\end{array}$ & $\begin{array}{l}0,39 \pm 0,12 \\
(p<0,001)\end{array}$ & $\begin{array}{c}0,46 \pm 0,11 \\
(p<0,001)\end{array}$ \\
\hline
\end{tabular}

\footnotetext{
r: coeficiente de correlação, ep: erro padrão. As demais abreviaturas podem ser visualizadas na Tabela 1

*: ajustado as covariáveis idade, sexo, idade ${ }^{2}$, idade ${ }^{3}$, idade $\times$ sexo, idade ${ }^{2} \times$ sexo e IMC

$t$ : ajustado as covariáveis idade, sexo, idade ${ }^{2}$, idade $\times$ sexo, idade ${ }^{2} \times$ sexo e IMC

キ: ajustado as covariáveis idade, sexo, idade ${ }^{2}$, idade $^{3}$, idade $\times \operatorname{sexo}$, idade $^{2} \times \operatorname{sexo}$, idade $^{3} \times$ sexo e IMC
}

dos resultados com outros estudos, tendo em vista que os parâmetros avaliados foram distintos e também devido a diferença na idade dos descendentes. Em apenas uma pesquisa(18) os descendentes apresentaram a faixa etária semelhante à do presente estudo. Foi verificado que os indivíduos do sexo feminino ostentaram valores de $\mathrm{GC}_{\text {rel }}$ superiores, sendo a maior magnitude verificada entre os progenitores $(\approx 10 \%)$. Mesmo considerando as diferenças metodológicas supracitadas, os resultados encontrados, em outras pesquisas, na $\mathrm{GC}_{\text {rel }}(24,31) \mathrm{e}$

$\mathrm{GC}_{\mathrm{abs}}{ }^{(8,19,24)}$ foram análogos aos do presente estudo. Tal como referido anteriormente, os objectivos deste estudo foram identificar AgF e estimar a magnitude dos factores genéticos em diferentes indicadores da CC. Os valores encontrados indicam uma forte contribuição genética na expressão final destas características. Diversas pesquisas procuraram quantificar a influência que os factores genéticos possuem em diferentes fenótipos marcadores da CC. Alguns trabalhos utilizaram a BIA $(3,13,15,16,31)$, enquanto outros recorreram a técnicas mais sofisticadas como a $\operatorname{DEXA}^{(4,5,9,29)}$, a pesagem hidrostática(23,27) ou a tomografia axial computorizada $(8,19,24)$.

$\mathrm{Na}$ presente investigação os fenótipos relacionados com a quantidade e distribuição de GC apresentaram estimativas de $h^{2}$ entre 0,35 a 0,43. Das investiga- ções revistas, somente quatro ${ }^{(4,5,9,20)}$ providenciaram estimativas de $\mathrm{h}^{2}$ para a GT, com os resultados situados entre $31^{(4,5)}$ e $64 \%^{(9)}$. Para a GM apenas o estudo de Pérusse et al.(20) salientou que a $\mathrm{h}^{2}$ foi igual a $34 \%$ da sua variação total. Já na $\mathrm{GC}_{\text {rel }}$, os resultados mostraram valores de $h^{2}$ entre $47^{(13)}$ e $76 \%$ (27). Para a quantidade de $\mathrm{GC}_{\text {abs }}$ a contribuição relativa dos factores genéticos é um pouco mais ampla, entre $33^{(4,5)}$ e $72 \%^{(9)}$. De uma maneira geral, os resultados vão de encontro às estimativas referidas em pesquisas realizadas em diversos países. O único fenótipo mostrando um resultado discordante e inferior aos demais referenciados na literatura foi a quantidade de $\mathrm{GC}_{\text {rel }}\left(0,37\right.$ vs $\left.0,47^{(13)}-0,76^{(27)}\right)$.

De maneira original, Pérusse et al.(20) verificaram a influência do exercício aeróbio nas estimativas de $h^{2}$ de algumas componentes da CC (GT, GM, somatório de dobras cutâneas - $\Sigma \mathrm{DC}$ e o rácio entre as $\mathrm{DC}$ do tronco e membros - RTM). Os resultados demonstraram que no início do estudo (quando os indivíduos eram sedentários) os fenótipos apresentaram estimativas de $h^{2}$ moderadas (GT $h^{2}=0,36, \mathrm{GM} \mathrm{h}^{2}=0,34$, $\Sigma \mathrm{DC} \mathrm{h}^{2}=0,31$ e RTM $\left.\mathrm{h}^{2}=0,50\right)$. Após 20 semanas de treino aeróbio, todas as estimativas de $\mathrm{h}^{2}$ reduziram $\left(\mathrm{GT} \mathrm{h}^{2}=0,21, \mathrm{GM} \mathrm{h}^{2}=0,15, \Sigma \mathrm{DC} \mathrm{h}^{2}=0,15\right.$ e RTM $\left.\mathrm{h}^{2}=0,14\right)$. De acordo com os resultados, pode inferirse uma forte influência do treino aeróbio na manifes- 
tação final destes fenótipos, bem como a sua dependência à variação genotípica entre famílias.

Para os fenótipos que expressam a MIG e a MM as estimativas de $\mathrm{h}^{2}$ encontradas foram de 0,35, a 0,46. Para a MIG os estudos consultados $(4,9,13,16,23)$ apresentaram $h^{2}$ entre $0,40^{(4)}$ a $0,72^{(13)}$. Hsu et al.(9) verificaram ainda que a MIG nos membros, apresentou uma componente genética de $67 \%$. Contudo, apesar da reconhecida importância da quantidade de MM em termos biológicos, não foi encontrada nenhuma evidência que tenha verificado a influência genética sobre a $\mathrm{MM}_{\text {rel }}$ e $\mathrm{MM}_{\mathrm{abs}}$. Não obstante, os resultados encontrados fornecem indícios que os factores genéticos exercem uma importante e significativa responsabilidade na manifestação final de ambas as características, sendo a maior, evidenciada pela $\mathrm{MM}_{\text {abs }}$.

$A h^{2}$ quantifica a proporção da variância total de um fenótipo que é atribuída aos factores genéticos em detrimento dos factores ambientais no seio das famílias(5). Sendo uma estimativa populacional existe algum cuidado na sua interpretação e tentativa de generalização. Hsu et al.(9) e North et al.(16) ressaltam alguns factores que reclamam atenção na atribuição de significado ao seu resultado: (1) diferença na magnitude das amostras; (2) aspectos étnicos e culturais inerentes a cada população; (3) diferentes designs das pesquisas; (4) ajustamento para covariáveis e procedimento de cálculos. Butte et al.(5) salientam ainda a necessidade de se ter atenção aos aspectos ambientais, que podem ser distintos quando os descendentes são crianças e/ou jovens se comparados aos adultos.

As correlações entre graus de parentesco pode auxiliar na interpretação das estimativas de $\mathrm{h}^{2}$ encontradas. Foram encontrados cinco estudos onde os autores calcularam as correlações para a GC(18,24,28,29,31); contudo, apenas num os autores calcularam as correlações para a $\mathrm{GT}$, a $\mathrm{GM}^{(20)}$ e a MIG(29). Na relação entre cônjuges para a GC, os resultados apresentaram valores de $r$ entre $-0,14$ a 0,16 . Savard et al.(28) e Park et al.(18) encontraram correlações entre 0,00 e 0,17 , respectivamente. Na relação progenitores-descendentes, os valores de $r$ se situaram entre $-0,04$ e 0,45 . Park et al.(18) e Rice et al.(24) encontraram valores de certa maneira semelhantes, onde $0,06 \leq r$ $\leq 0,33$. Na relação entre irmãos os resultados foram moderados (irmã-irmã: $r=0,12$ e irmão-irmão: $r=$
$0,65)$. Estes valores são corroborados por Savard et al. (28) e Rice et al. (24) $(0,19 \leq r \leq 0,46$, respectivamente). As correlações foram baixas para a GT em todos os graus de parentesco (cônjuges $r=-0,15$, progenitores-descendentes $-0,04 \leq r \leq 0,23$ e irmãos $0,04 \leq r$ $\leq 0,22$ ). Estes resultados foram semelhantes aos encontrados por Pérusse et al.(20) (cônjuges: $r=0,15$, progenitores-descendentes: $0,11 \leq r \leq 0,27$ e irmãos: $0,14 \leq r \leq 0,25$ ). Para a GM as correlações verificadas, em ambos os estudos, são semelhantes em todos os graus de parentesco, quando comparada a GT. O único estudo que apresentou correlações para a MIG foi o de Treuth et al.(29). Os autores procuraram verificar $\mathrm{AgF}$ entre progenitores-descendentes em alguns fenótipos da CC, em famílias onde os descendentes eram meninas pré-puberes. Os resultados foram de $r=0,37$ e $r=0,48$ para as relações entre paifilha e mãe-filha, respectivamente. Os resultados encontrados na presente investigação foram inferiores (pai-filha $r=-0,04$ e mãe-filha $r=0,24$ ).

De uma maneira geral, para os sete fenótipos avaliados, os resultados referem uma menor AgF entre cônjuges $(-0,04 \leq r \leq 0,23)$ quando comparado aos progenitores-descendentes $(-0,04 \leq r \leq 0,48)$ e irmãos $(0,01 \leq r \leq 0,65)$.

É bem provável que se verificasse alguma alteração dos valores de correlação entre familiares e estimativas de heritabilidade dos diferentes indicadores da CC se fosse considerada informação suplementar relativa a hábitos nutricionais, níveis e padrões de actividade física. Convém referir que dispomos de parte desta informação que está a ser, actualmente, objecto de estudo a que se associam indicadores da síndrome metabólica.

Os resultados das pesquisas de AgF salientam a contribuição dos factores genéticos em diferentes fenótipos da CC. Uma área de estudo sequencial em Epidemiologia Genética tem o propósito de identificar regiões, em diferentes cromossomas, que possam albergar os genes responsáveis pela variabilidade destes fenótipos (i.e., estudos de Linkage). A título de ilustração, no último "Update" do The Human Obesity Gene Map, Rankinen et al.(22) referenciaram estudos com sinais de linkage significativo em alguns fenótipos da CC, como por exemplo, a $\mathrm{GC}_{\text {rel }}$ (D2S2739/2p16 e TNF/6p21.3), GC $_{\text {abs }}$ (D14S283/14q11.2 e LEP/7q31.3), MIG (LEPR- 
Q223R/1p31.2 e D15S652/15q26.1), gordura visceral (IGF1/12q23.3), entre outros, bem como referenciaram alguns genes candidatos responsáveis por variações bem díspares nalguns fenótipos descritores da CC. São estas as etapas futuras da pesquisa: (1) uso de genes candidatos em estudos clássicos de caso-controlo ou de famílias; (2) recurso a microarrays; (3) estudos funcionais com animais, sobretudo com knock-out de genes relevantes; (4) pesquisa de interacção genes $\mathrm{x}$ ambiente, sendo que este corresponde a condições distintas e controladas de actividade física/exercício; (5) estudos relativos aos mecanismos fisiológicos associados às respostas dos diferentes componentes da massa corporal a situações extremas de baixo peso e obesidade em resposta a alteração das condições nutricionais e de exercício físico.

\section{CONCLUSÕES}

De acordo com os resultados encontrados conclui-se que: (1) os factores genéticos foram responsáveis por 35 a $46 \%$ da variação total dos sete fenótipos avaliados pela BIA e (2) estes resultados indicam uma forte $\mathrm{AgF}$ na CC nesta amostra de famílias nucleares portuguesas. Com base nas correlações efectuadas, verificou-se um padrão distinto de $\mathrm{AgF}$ entre os pares de indivíduos, sendo a maior semelhança verificada entre irmãos.

\section{AGRADECIMENTOS}

Gostaríamos de agradecer: (1) as sugestões dos revisores que melhoraram aspectos do texto; (2) a colaboração de Amélia Martins, João Vinagre, Rita Miranda, Ramon Lima, Sónia Vidal, Sílvio Saranga, Leonardo Nhantumbo, Simonete Silva e Renata Karine na recolha da informação.

\section{CORRESPONDÊNCIA}

\section{José António Ribeiro Maia}

Universidade do Porto - Faculdade de Desporto. Laboratório de Cineantropometria e Gabinete de Estatística Aplicada

Rua Dr. Plácido Costa, 91 - 4200-450

Porto - Portugal.

E-mail: jmaia@fade.up.pt 


\section{REFERÊNCIAS}

1. American College of Sports Medicine (2003). Diretrizes do ACSM para os testes de esforço e sua prescrição, Rio de Janeiro: Guanabara Koogan.

2. American Heart Association (2007). Heart Disease and Stroke Statistics - 2007 Update. A Report From the American Heart Association Statistics Committee and Stroke Statistics Subcommittee. Circulation 115 1-101.

3. Bayoumi RA, Al-Yahyaee SA, Albarwani SA, Rizvi SG, AlHadabi S, Al-Ubaidi FF, Al-Hinai AT, Al-Kindi MN, Adnan HT, Al-Barwany HS, Comuzzie AG, Cai G, LopezAlvarenga JC, Hassan MO (2007). Heritability of determinants of the metabolic syndrome among healthy Arabs of the Oman family study. Obesity 15(3):551-556.

4. Butte NF, Cai G, Cole SA, Comuzzie AG (2006). Viva la Familia Study: genetic and environmental contributions to childhood obesity and its comorbidities in the Hispanic population. Am J Clin Nutr 84(3):646-654.

5. Butte NF, Comuzzie AG, Cole SA, Mehta NR, Cai G, Tejero M, Bastarrachea R, Smith EO (2005). Quantitative genetic analysis of the metabolic syndrome in Hispanic children. Pediatr Res 58(6):1243-1248.

6. Campos MA, Maia JAR, Seabra A, Silva RG, Lopes VP, Freitas DL (2007). Actividade física e componentes da síndrome metabólica. Um estudo em famílias açorianas, Porto: Direcção Regional do Desporto da Região Autónoma dos Açores e FADE-UP.

7. Heyward V (2001). ASEP methods recommendation: body composition assesment. J Exerc Physiol 4(4):1-12.

8. Hong Y, Rice T, Gagnon J, Despres JP, Nadeau A, Perusse L, Bouchard C, Leon AS, Skinner JS, Wilmore JH, Rao DC (1998). Familial clustering of insulin and abdominal visceral fat: the HERITAGE Family Study. J Clin Endocrinol Metab 83(12):4239-4245.

9. Hsu FC, Lenchik L, Nicklas BJ, Lohman K, Register TC, Mychaleckyj J, Langefeld CD, Freedman BI, Bowden DW,Carr JJ (2005). Heritability of body composition measured by DXA in the diabetes heart study. Obes Res 13(2):312-319.

10. Hunt MS, Katzmarzyk PT, Perusse L, Rice T, Rao DC, Bouchard C (2002). Familial resemblance of 7-year changes in body mass and adiposity. Obes Res 10(6):507-517.

11. Katzmarzyk PT, Malina RM, Perusse L, Rice T, Province MA, Rao DC, Bouchard C (2000). Familial resemblance in fatness and fat distribution. Am J Human Biol 12(3):395-404.

12. Lohman TG, Roche AF, Martorell R (1988). Antropometric standardization reference manual. Champaign.

13. Luke A, Guo X, Adeyemo AA, Wilks R, Forrester T, Lowe W, Comuzzie AG, Martin LJ, Zhu X, Rotimi CN, Cooper RS (2001). Heritability of obesity-related traits among Nigerians, Jamaicans and US black people. Int J Obes Relat Metab Disord 25(7):1034-1041.

14. Mattsson S, Thomas BJ (2006). Development of methods for body composition studies. Phys Med Biol 51 (13):203228.

15. Mills GW, Avery PJ, McCarthy MI, Hattersley AT, Levy JC, Hitman GA, Sampson M, Walker M (2004). Heritability estimates for beta cell function and features of the insulin resistance syndrome in UK families with an increased susceptibility to type 2 diabetes. Diabetologia 47(4):732-738.

16. North KE, Howard BV, Welty TK, Best LG, Lee ET, Yeh JL, Fabsitz RR, Roman MJ, MacCluer JW (2003). Genetic and environmental contributions to cardiovascular disease risk in American Indians: the strong heart family study. Am J Epidemiol 157(4):303-314.

17. Ogden CL, Carroll MD, Curtin LR, McDowell MA, Tabak CJ, Flegal KM (2006). Prevalence of overweight and obesity in the United States, 1999-2004. Jama 295 (13):15491555.

18. Park HS, Yim KS, Cho SI (2004). Gender differences in familial aggregation of obesity-related phenotypes and dietary intake patterns in Korean families. Ann Epidemiol 14(7):486-491.

19. Perusse L, Despres JP, Lemieux S, Rice T, Rao DC, Bouchard C (1996). Familial aggregation of abdominal visceral fat level: results from the Quebec family study. Metabolism 45(3):378-382.

20. Perusse L, Rice T, Province MA, Gagnon J, Leon AS, Skinner JS, Wilmore JH, Rao DC, Bouchard C (2000). Familial aggregation of amount and distribution of subcutaneous fat and their responses to exercise training in the HERITAGE family study. Obes Res 8(2):140-150.

21. Pietrobelli A, Rubiano F, St-Onge MP, Heymsfield SB (2004). New bioimpedance analysis system: improved phenotyping with whole-body analysis. Eur J Clin Nutr 58(11):1479-1484.

22. Rankinen T, Zuberi A, Chagnon YC, Weisnagel SJ, Argyropoulos G, Walts B, Perusse L, Bouchard C (2006). The human obesity gene map: the 2005 update. Obesity 14(4):529-644.

23. Rice T, Daw EW, Gagnon J, Bouchard C, Leon AS, Skinner JS, Wilmore JH, Rao DC (1997). Familial resemblance for body composition measures: the HERITAGE Family Study. Obes Res 5(6):557-562.

24. Rice T, Despres JP, Daw EW, Gagnon J, Borecki IB, Perusse L, Leon AS, Skinner JS, Wilmore JH, Rao DC, Bouchard C (1997). Familial resemblance for abdominal visceral fat: the HERITAGE family study. Int J Obes Relat Metab Disord 21(11):1024-1031.

25. Rice T, Perusse L, Bouchard C, Rao DC (1999). Familial aggregation of body mass index and subcutaneous fat measures in the longitudinal Quebec family study. Genet Epidemiol 16(3):316-334.

26. S.A.G.E. 5.3 (2005). Statistical Analysis for Genetic Epidemiology http://darwin.cwru.edu/sage/.

27. Sakul H, Pratley R, Cardon L, Ravussin E, Mott D, Bogardus C (1997). Familiality of physical and metabolic characteristics that predict the development of non-insulin-dependent diabetes mellitus in Pima Indians. Am J Hum Genet 60(3):651-656.

28. Savard R, Bouchard C, Leblanc C, Tremblay A (1983). Familial resemblance in fatness indicators. Ann Hum Biol 10(2):111-118.

29. Treuth MS, Butte NF, Ellis KJ, Martin LJ, Comuzzie AG (2001). Familial resemblance of body composition in prepubertal girls and their biological parents. Am J Clin Nutr 74(4):529-533.

30. Wigginton JE, Abecasis GR (2005). PEDSTATS: descriptive statistics, graphics and quality assessment for gene mapping data. Bioinformatics 21(16):3445-3447.

31. Wu DM, Hong Y, Sun CA, Sung PK, Rao DC, Chu NF (2003). Familial resemblance of adiposity-related parameters: results from a health check-up population in Taiwan. Eur J Epidemiol 18(3):221-226 